\title{
Projeto e implementação de uma rede neural artificial para detecção do mal-posicionamento rotacional de dedos em dispositivos de captura de impressões digitais multivista sem toque
}

\author{
Alternative Title: Design and implementation of an artificial neural network applied \\ to finger bad-positioning detection on touchless multiview fingerprints devices
}

\author{
Cauê Zaghetto \\ Prog. de Pós-graduação em \\ Sistemas Mecatrônicos \\ Universidade de Brasília \\ Brasília, Brasil \\ cauezag@aluno.unb.br
}

\author{
Luiz Henrique M. Aguiar \\ Prog. de Pós-graduação em \\ Sistemas Mecatrônicos \\ Universidade de Brasília \\ Brasília, Brasil \\ luizhenrique@aluno.unb.br

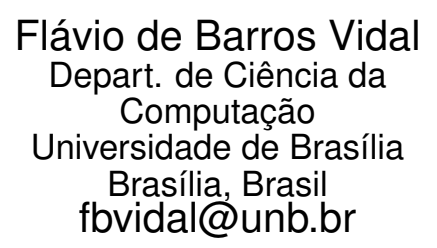
Alexandre Zaghetto
Depart. de Ciência da
Computação

Universidade de Brasília

Brasília, Brasil

alexandre@cic.unb.br

\section{RESUMO}

Este artigo apresenta um método baseado em Redes neurais artificiais que avalia o mal-posicionamento dos dedos, em função da rotação, em dispositivos de aquisição de impressões digitais multivista sem toque. O objetivo é determinar se o dedo está rotacionado ou não, uma vez que o bom posicionamento do dedo é mandatório para garantir altas taxas de correspondência de impressões digitais. Um conjunto de teste de 9000 imagens foi usado para treinar, validar e testar um classificador baseado em redes neurais artificiais multicamadas. Até o momento, não existe um método definitivo que abordou o problema de qualidade de impressões digitais em scanners que utilizem a tecnologia multivista sem toque e a deteç̧ão da rotação do dedo apresentada neste artigo é um dos passos que devem ser levados em conta se um futuro método automático para avaliação da qualidade de impressões digitais for considerado. Os resultados médios, mostram que: (a) o classificador identifica corretamente o mal-posicionamento em aproximadamente $94 \%$ dos casos; e (b) quando o mal-posicionamento é detectado, o ângulo de rotação é corretamente estimado em $90 \%$ dos casos.

Permission to make digital or hard copies of all or part of this work for personal or classroom use is granted without fee provided that copies are not made or distributed for profit or commercial advantage and that copies bear this notice and the full citation on the first page. To copy otherwise, to republish, to post on servers or to redistribute to lists, requires prior specific permission and/or a fee.

SBSI 2015, May 26th-29th, 2015, Goiânia, Goiás, Brazil

Copyright SBC 2015.

\section{Palavras-Chave}

impressão digital, multivista, sem toque, avaliação de qualidade.

\begin{abstract}
This paper presents a method based on Artificial Neural Network that evaluates the rotational bad-positioning of fingers on touchless multiview fingerprinting devices. The objective is to determine whether the finger is rotated or not, since a proper positioning of the finger is mandatory for high fingerprint matching rates. A test set of 9000 acquired images has being used to train, validate and test the proposed multilayer Artificial Neural Network classifier. To our knowledge, there is no definitive method that addressed the problem of fingerprint quality on touchless multiview scanners. The proposed finger rotation detection here presented is one of the steps that must be taken into account if a future automatic image quality assessment method is to be considered. Average results show that: (a) our classifier correctly identifies bad-positioning in approximately $94 \%$ of cases; and (b) if bad-positioning is detected, the rotation angle is correctly estimated in $90 \%$ evaluations.
\end{abstract}

\section{Categories and Subject Descriptors}

H.4 [Information Systems Applications]: Miscellaneous

\section{General Terms}

Fingerprint quality assessment

\section{Keywords}

fingerprint, multiview, touchless, quality assessment. 


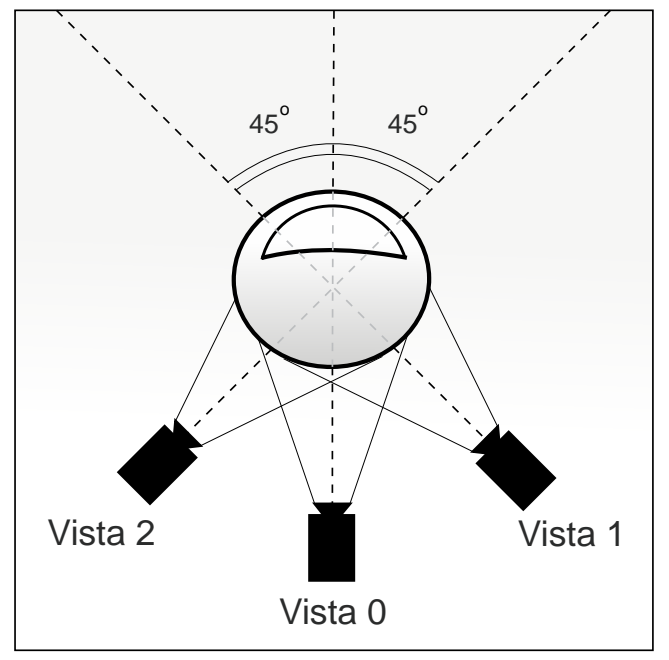

Figura 1: Descrição de um sistema de captura para impressão digital multivista sem toque, utilizando três câmeras (uma central e duas laterais) para captura da área central e lateral da impressão digital.

\section{INTRODUÇÃO}

A autenticação biométrica, ou simplesmente biometria, pode ser definida como a verificação ou reconhecimento automático de identidade de um indivíduo com base nas características fisiológicas e comportamentais [19]. Impressão digital, geometria da mão, voz, íris, face, escrita à mão e forma/tempo de digitação no teclado são exemplos de tais características. Diferentes sistemas biométricos requerem tecnologias específicas, dependendo do aspecto fisiológico(ou comportamental) que está sendo usado [18]. No entanto, a biometria baseada em impressão digital é hoje o sistema mais usado e reconhecido pela indústria e academia. Se comparado a outros parâmetros biométricos, a impressão digital é a mais popular e amplamente utilizado em todo o mundo. Uma grande parte de suas aplicações está associada à identificação civil e investigação criminal. Praticamente, todos os departamentos policiais [10] possuem um Sistema Automático de Identificação de Impressões Digitais (AFIS) [9]. Apesar de sua maturidade e ampla divulgação, acredita-se que os algoritmos de aquisição de impressão digital, processamento de imagens e de correspondência não estão no seu potencial pleno e definitivo [16].

Dessa forma, uma análise mais cuidadosa mostra que há muito espaço para melhorias não só para aprimorar o estado da arte dos algoritmos e processamento, mas também para mostrar novas e melhores formas de realizar a biometria. Nós descrevemos brevemente uma nova, mas já conhecida, tecnologia de aquisição de impressões digitais multivista sem toque, ilustrada na Figura 1. Esta resolve muitos problemas de scanners baseados em toque, mas é suscetível a novos. O problema mais fundamental desta tecnologia reside no fato de que é preciso garantir o correto posicionamento do dedo em relação à câmara de aquisição. Este problema surge a partir do fato de que no processo de aquisição utilizando um dispositivo sem uma superfície de toque, os dedos estão sujeitos a mais graus de liberdade. Até onde se sabe, não existe um método definitivo, que aborda o problema da qualidade da impressão digital para scanners multivistas sem toque.
Portanto, neste artigo apresentamos um algoritmo que indica se o dedo foi colocado corretamente ou não, devido à possibilidade de mal-posicionamento rotacional do mesmo. Este é o primeiro passo para a definição de um método de avaliação de qualidade mais completo, que pode orientar o processo de aquisição de impressão digitais sem toque no futuro. Desta feita, as informações do processo identificação de impressões digitais e metodologia proposta para se alcançar os resultados são descritos na Seção 2 e 3, respectivamente. Os resultados são apresentados e discutidos na Seção 4. Finalizando na Seção 5, em que as conclusões e os trabalhos futuros são apresentados.

\section{IDENTIFICAÇÃO DE IMPRESSÕES DI- GITAIS}

Em uma forma simplificada, o processo de identificação de impressões digitais pode ser subdividido em quatro subprocessos: (1) Captura da imagem; (2) Pré-processamento; (3) Extração de Características; e; (4) Correspondência das características. Entre as diversas classificações que podem ser realizadas na identificação de impressões digitais, a maioria faz do uso de técnicas de capturas da imagem com a necessidade do toque entre o dedo do usuário e o dispositivo de captura utilizado. Atualmente o processo de captura é dividido de acordo com o tipo de tecnologia: com ou sem toque do dedo (i. e. touchless) do usuário no dispositivo. Se por um lado, sistemas em que o toque no sensor se faz necessário, já alcançaram um nível de maturidade, por outro, sistemas que utilizam a tecnologia sem toque no sensor ainda estão nos seus primórdios, permitindo ainda diversos avanços no processo de identificação biométrica, a partir da impressão digital.

\subsection{Impressões digitais com toque}

Atualmente diversas aplicações nas áreas forenses, civis e comerciais [1] fazem uso de impressões digitais. Nestes sistemas, a autenticação a partir do uso de imagens digitais das impressões digitais, permitem uma descrição real dos dedos, aumentando a confiabilidade do sistema. Desta feita, o processo de aquisição se torna um passo crucial do sistema, interferindo diretamente no desempenho geral do processo de identificação. A maioria dos sistemas atuais empregam tecnologias baseadas no toque do dedo do usuário com o sensor de captura, demandando ao usuário pressionar o dedo contra sua superfície.

Dentre os diversos problemas relacionados ao uso deste tipo de tecnologia, podemos citar as distorções e inconsistências causadas, durante o processo de captura, pela elasticidade da pele. A qualidade da impressão digital obtida pode ser também influenciada pela sujeira, suor, secura excessiva, umidade do ar, temperatura e impressões digitais danificadas (cicatrizes). Também as variações no posicionamento do dedo em cada captura, pode ocasionar em amostras diferentes de imagens capturadas para o mesmo dedo. Em algumas situações, estas desvantagens apresentadas em [8], impõem a necessidade de operadores extremamente especializados e treinados, requerendo maior quantidade de tentativas, e proporcionalmente elevando o tempo necessário para um processo de captura para todos os dedos. Por exemplo, para o cadastramento de grandes populações, como o cadastramento biométrico eleitoral realizado em 2013 e 2014 no Brasil, demandam de muito tempo para que as impressões 


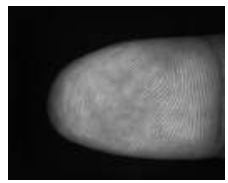

(a)

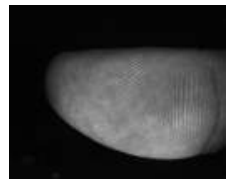

(b)

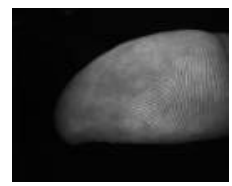

(c)
Figura 2: Exemplo de impressões digitais capturadas $(1024 \times 1280$ pixels, 8 bits $/$ pixels $)$ capturadas por um sistema sem toque com três câmeras: (a) Vista 0; (b) Vista 1; and (c) Vista 2.

digitais capturadas tenham a qualidade necessária para o processo de identificação.

Com o passar dos anos, diversos algoritmos foram propostos para compensar essas limitações das tecnologias baseadas em toque, sendo que em alguns, estas melhorias geram gargalos para melhorias futuras na qualidade da imagem digital proveniente destas impressões digitais capturadas.

\subsection{Impressões digitais sem toque}

Atualmente, diversas soluções baseadas na captura de impressões digitais sem toque tem sido desenvolvidas. Todas estas para colaborar no processo de melhoria dos problemas intrínsecos dos sistemas, em que utilizam o toque no sensor durante o processo de captura $[18,11,12,13]$. A idéia básica de sistemas sem toque é atacar o problema de qualidade da amostra em seu nível fundamental: durante o processo de captura. No caso da utilização destes sistemas, não existe a necessidade do usuário em pressionar o dedo contra o sensor, evitando os problemas listados anteriormente, e ainda, não dependendo da utilização de algoritmos para a melhoria da qualidade da amostra capturada para compensar os problemas causados pela elasticidade da pele humana.

Dentre as soluções tecnológicas disponíveis, a empresa suíça TBS $^{1}$ desenvolveu um dispositivo [17] que utiliza uma metodologia promissora. Este dispositivo combina técnicas baseada na captura da reflexão da imagem do dedo a ser capturado, com um sistema de três câmeras sincronizadas em posicionamentos diferentes (múltiplas vistas). Uma câmera é posicionada para capturar a imagem proveniente do posicionamento normal ao dedo, em que a parte principal da impressão digital está localizada (deltas e bifurcações), a partir de uma câmera central. As outras duas câmeras são posicionadas a $45^{\circ}$, sendo uma no sentido horário $(\mathrm{SH})$ e a outra no sentido anti-horário(SAH). A partir desta abordagem, é garantida a captura sobreposta de todas as áreas da impressão digital capturada, como apresentado na Figura 1. A imagem final é obtida a partir do mosaico formado das três imagens utilizando algoritmos de alinhamento [14]. Nas Figuras 1 e 2, são apresentadas uma representação esquemática de um sistema de captura sem toque utilizando múltiplas vistas e imagens capturadas por este tipo de dispositivo, respectivamente.

\subsection{Avaliação da qualidade de impressões di- gitais}

O desempenho e interoperabilidade dos sistemas biométricos dependem da qualidade do sinal adquirido [2]. Entretanto, uma metodologia para a estimação da qualidade

\footnotetext{
${ }^{1}$ http://www.tbsinc.com/
}

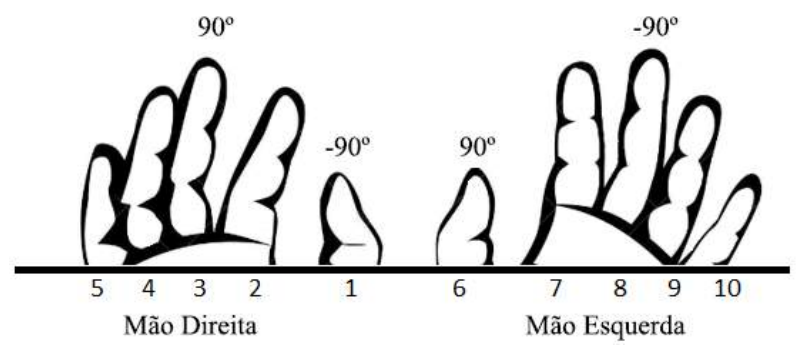

Figura 3: Identificação dos dedos e possíveis direções de rotações.

das amostras biométricas coletadas são de fundamental importância, principalmente para se obter elevados índices de acerto.

Existe uma grande variedade de tecnologias que realizam a captura da impressão digital. Mesmo com os avanços e aprimoramentos muitos desses sistemas de capturas falham no processo de obtenção de imagens com boa qualidade. Alguns dispositivos de captura podem ser melhores do que outros, sendo até imunes a fatores que afetam diretamente a qualidade da imagem produzida, mas o problema em se obter imagens com boa qualidade ainda permitem avanços. Em trabalhos recentes, vários problemas que afetam a qualidade da impressão digital (como por exemplo toques com o sensor inconsistentes, não-uniformes e irreprodutíveis [15]) propõem técnicas que resolvem estes problemas $([6,4,5])$. Sendo assim, a análise da avaliação da qualidade são originárias dos mesmos princípios dos sistemas baseados na captura com o toque do dedo do usuário no sensor, estas propostas pelo National Institute of Standards and Technology (NIST) [20], fazendo necessária na utilização de sistemas sem toque se adequem aos mesmos níveis de exigências do sistema com toque.

\section{METODOLOGIA PROPOSTA}

Uma vez que o objetivo é avaliar o mal-posicionamento dos dedos em dispositivos de impressão digital sem toque, o primeiro passo é criar um conjunto de testes. As amostras foram adquiridas a partir de 20 voluntários que cederam suas impressões digitais capturadas por um dispositivo com características gerais semelhantes às descritas na Figura 1. Amostras provenientes de todos os dedos de ambas as mãos foram coletadas. Aqui, a amostra é definida como um conjunto de três imagens (vista 0,1 e 2 ) de cada dedo, como mostrado na Figura 2. Todos os dedos (10 dedos) geram 15 amostras, o que perfaz um total de 45 imagens por dedo. Portanto, o nosso conjunto de teste é composto por $20 \times 10 \times 15 \times 3=9000$ imagens. As 15 amostras de cada dedo são distribuídas da seguinte forma:

- 5 amostras não rotacionadas;

- 5 amostras rotacionadas em $180^{\circ}$; e

- 5 amostras rotacionadas em $90^{\circ}$ sentido horário (SH) ou sentido anti-horário ( $\mathrm{SAH}$ ), dependendo da mão (esquerda ou direita).

Quatro dedos da mão direita $(2,3,4$ e 5$)$ e um dedo da mão esquerda (6) foram rotacionados no sentido horário. Quatro dedos da mão esquerda $(7,8,9$ e 10) e um 


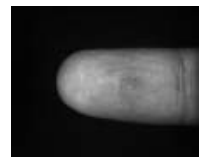

(a)

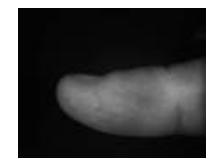

(b)

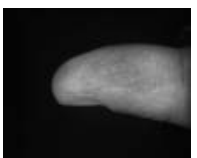

(c)

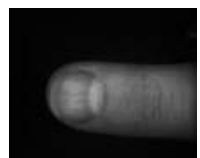

(d)

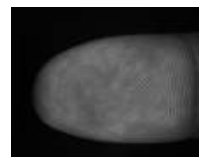

(a)

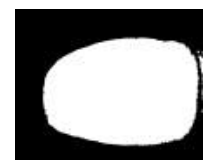

(b)

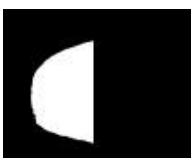

(c)

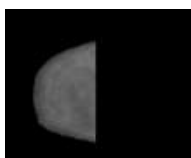

(d)
Figura 4: Efeito de diferentes ângulos de rotação na Vista 0: (a) não-rotacionado; (b) $90^{\circ} \mathbf{S H}$; (c) $90^{\circ}$ $\mathrm{SAH}$; and (d) rotacionado em $180^{\circ}$.

dedo da mão direita (1) foram rotacionados no sentido anti-horário. Os polegares foram rotacionados no sentido oposto, considerando os outros dedos da mesma mão, devido a razões ergonômicas. A Figura 3 identifica cada dedo e os respectivos sentidos de rotação. O efeito dos diferentes sentidos de rotação sobre a vista 0 é exemplificada na Figura 4. Consequentemente, o conjunto de testes é caracterizado por 500 amostras de dedos rotacionados em $90^{\circ}$ sentido horário, 500 amostras de dedos rotacionados em $90^{\circ}$ sentido anti-horário, 1.000 amostras de dedos rotacionados em $180^{\circ}$ e 1000 amostras de dedos não rotacionados, o que perfaz um total de 3.000 amostras.

O próximo passo é definir as métricas que serão utilizados como parâmetros para o classificador. A primeira métrica é a média da área útil em cada vista $\left(m_{0}, m_{1}\right.$ e $\left.m_{2}\right)$. A segunda métrica é a variância destas mesmas áreas úteis $\left(\sigma_{1}, \sigma_{2}\right.$ e $\left.\sigma_{3}\right)$. Nós assumimos que a luminância média e a textura irão variar dependendo da porção do dedo a ser fotografado. A unha produz, sem dúvida, diferentes padrões de reflexão em comparação à parte do dedo que contém o núcleo e o delta.

Para calcular os valores destas duas métricas, primeiro uma uma área útil deve ser delimitada. Inicialmente, aplicamos um processo de binarização com o método de Otsu [21]. Uma vez que o fundo é muito mais escuro do que o primeiro plano, o processo retorna uma segmentação do dedo muito precisa. Em seguida, o elemento de imagem branco mais à esquerda é considerado como a ponta de um dedo. Considerando-se que o comprimento do dedo $L$ em pixels pode ser estimado como o número de colunas entre a ponta do dedo coluna $f_{c}$ e a última coluna da imagem, a região de interesse (ROI) é definida como todos os pixels brancos que encontram-se entre $f_{c}$ e $f_{c}+L / 2$. A única garantia é uma área significativa do dedo estar representada dentro desta região. Pelo menos a unha deve ser incluída. A Figura 5 ilustra como a região de interesse é capturada. Além das métricas descritas anteriormente, as razões entre a largura dos dedos projetadas em cada vista também são consideradas. Como mostrado na Figura 6 as razões entre as larguras das vistas $1\left(w_{1}\right)$ e $2\left(w_{2}\right)$ em relação à vista $0\left(w_{0}\right)$ podem ser utilizadas como um parâmetro discriminativo.

Uma vez que as métricas são apresentadas, o vetor de características $\mathbf{X}$, que vai ser entrada para o classificador, pode ser definido. Este vetor é composto por 6 elementos, de $x_{1}$ a $x_{6}$, descrito como se segue:

$$
\mathbf{X}=\left[\begin{array}{ccc}
m_{1} / m_{0} & m_{2} / m_{0} & \sigma_{1} / \sigma_{0} \\
\sigma_{2} / \sigma_{0} & w_{1} / w_{0} & w_{2} / w_{0}
\end{array}\right]
$$

Com intuito de detectar quando o dedo está, ou não, de-
Figura 5: Recuperação da região de interesse (Vista 0): (a) Imagem original; (b) Resultado da binarização; (c) Seleção da Máscara; e (d) área selecionada para o cálculo da média e variância. O mesmo procedimento é aplicado para as vistas 1 e 2 .

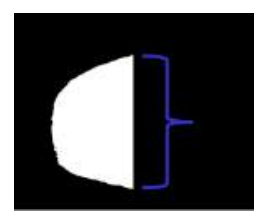

(a)

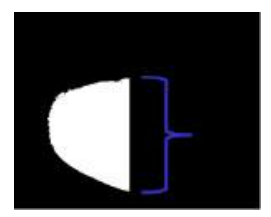

(b)

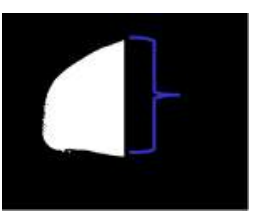

(c)
Figura 6: Dedo projetado em diferentes vistas de uma amostra: (a) Projeção da Vista 0; (b) Projeção da Vista 1; e (c) Projeção da Vista 2. Razões entre as larguras das Vistas 1 e 2 em relação à Vista 0 também podem ser usadas como um parâmetro discriminativo no vetor de características.

vidamente posicionado durante a aquisição biométrica, uma Rede Neural Artificial é proposta. O classificador é um Perceptron Multicamada [7] treinado com o algoritmo de Levenberg-Marquardt [22] que tem quatro camadas: (a) uma camada de entrada, onde nenhum cálculo é realizado; (b) duas camadas intermediárias; e (c) uma camada de saída. A primeira camada simplesmente insere vetor $\mathbf{X}$ para a rede neural proposta. A primeira e a segunda camadas ocultas têm 20 e 5 neurônios, respectivamente. A camada de saída tem um único neurônio. Exceto para a camada de entrada, todos os neurônios têm a função de ativação Tangente Hiperbólica. Sabe-se que as redes neurais sem realimentação (feedforward) com apenas uma camada escondida podem aproximar satisfatoriamente qualquer função com um número finito de descontinuidades arbitrárias [3]. No entanto, após testes exaustivos, observamos em nossos experimentos que melhores desempenhos foram alcançados com duas camadas ocultas. A arquitetura proposta é resumida na Figura 7 .

Em nossos experimentos, dois cenários diferentes foram considerados. Em um primeiro cenário, o classificador apenas indica se existe um mal-posicionamento rotacional do dedo ou não. Em um segundo, caso o classificador tenha detectado mal-posicionamento rotacional, também detecta o ângulo de rotação $\left(90^{\circ}\right.$ horário, $90^{\circ}$ sentido anti-horário e $\left.180^{\circ}\right)$.

$$
\alpha(T)= \begin{cases}180^{\circ}, & \text { se }-1 \leq T \leq-0.65 \\ 90 \mathrm{SAH}, & \text { se }-0.65<T \leq 0.05 \\ 90 \mathrm{SH}, & \text { se } 0.05<T \leq 0.70 \\ 0^{\circ} \text { (Não-rotacionado), } & \text { se } 0.70<T \leq 1\end{cases}
$$




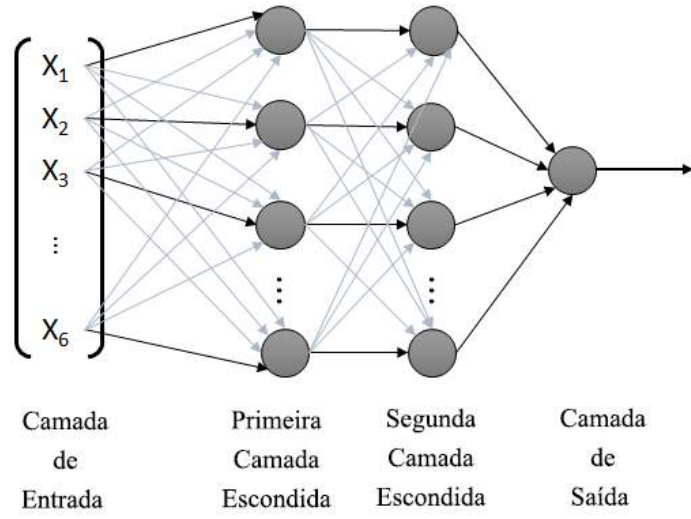

Figura 7: Arquitetura proposta para a rede neural artificial utilizada: camada de entrada com 6 neurônios, de $x_{1}$ a $x_{6}$; primeira camada oculta com 20 neurônios; segunda camada oculta com 5 neurônios; e camada de saída com 1 neurônio. Todos os neurônios possuem como função de ativação a tangente hiperbólica.

No primeiro cenário, o neurônio da camada de saída retorna $T=-1$ (no caso de rotação) ou $T=1$ (no caso de correto posicionamento). Quanto ao segundo cenário, os valores de saída são quantificados em um determinado ângulo de rotação $\alpha$, de acordo com Eq. 2 .

O primeiro conjunto de testes usado em nossos experimentos é composto por todas as amostras disponíveis: 1000 dedos não rotacionados (20 indivíduos, 10 dedos diferentes por indivíduo, cinco amostras por dedo) e 2.000 dedos rotacionados (20 indivíduos, 10 dedos diferentes por indivíduo, 10 amostras por dedo: todos os dedos rotacionados por $180^{\circ}$, e por $90^{\circ} \mathrm{SH}$ ou $90^{\circ} \mathrm{SAH}$, dependendo de cada dedo). Cerca de $1 / 3$ de cada classe, não rodados e rodados (por $180^{\circ}, 90^{\circ} \mathrm{SH}$ ou $90^{\circ} \mathrm{SAH}$ ) foi utilizado para compor o conjunto de treinamento, validação e teste da rede neural. O conjunto de treinamento é usado para atualizar as sinapses e biases da rede, o conjunto de validação é usado para monitorar o processo de formação e evitar super-ajuste. $\mathrm{O}$ conjunto de teste é utilizado para avaliar o desempenho da rede e não é utilizado durante o treinamento.

Um caso especial também foi investigado. Uma vez que o polegar é um dedo largamente utilizado na identificação biométrica, os dois cenários descritos acima também são aplicados para este dedo apenas. Neste caso, o subconjunto de ensaio é composto por 600 amostras de polegares: 200 representando a classe de dedos não rotacionados e 400 representando a classe de dedos rotacionados. Também neste caso, cerca de 1/3 de cada classe foi distribuída entre o treinamento, validação e conjunto de teste.

\section{RESULTADOS EXPERIMENTAIS}

Os testes foram divididos em quatro categorias:

- Rotacionado $\times$ Não rotacionado: detecção de rotação para todos dedos; e;

- Rotacionado × Não rotacionado: detecção de rotação dos polegares.
Tabela 1: Resultado da detecção de todos os dedos: Rotacionado $\times$ Não-rotacionado.

\begin{tabular}{|c|c|c|c|}
\hline \multirow{4}{*}{ 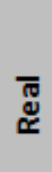 } & \multicolumn{3}{|c|}{ Predição } \\
\hline & \multirow{3}{*}{$\begin{array}{l}\text { Não rot. } \\
\text { Rot. }\end{array}$} & Não rot. & Rot. \\
\hline & & $86.90 \%$ & $13.10 \%$ \\
\hline & & $7.00 \%$ & $93.00 \%$ \\
\hline
\end{tabular}

Tabela 2: Resultado da detecção do polegar: Rotacionado $\times$ Não-rotacionado.

\begin{tabular}{|c|c|c|c|}
\hline \multirow{4}{*}{$\begin{array}{l}\overline{\text { Tु }} \\
\text { व }\end{array}$} & \multicolumn{3}{|c|}{ Predição } \\
\hline & \multirow{3}{*}{$\begin{array}{l}\text { Não rot. } \\
\text { Rot. }\end{array}$} & Não rot. & Rot. \\
\hline & & $96.50 \%$ & $3.50 \%$ \\
\hline & & $2.75 \%$ & $97.25 \%$ \\
\hline
\end{tabular}

- Detecção do ângulo de rotação de todos os dedos; e;

- Detecção do ângulo de rotação dos polegares.

As Tabelas 1, 2, 3 e 4 apresentam os resultados para cada categoria avaliada, descrita anteriormente. As porcentagens apresentadas nas Tabelas 1 e 2 indicam as taxas em que um dedo rotacionado ou não é corretamente classificado. As Tabelas 3 e 4 indicam as taxas em que casos para ângulos de rotação reais são classificados de acordo com a rotação predita. As diagonais representam os valores de verdadeiros positivos e são descritos nos gráficos de barras das Figuras 8(a) e (b).

No primeiro cenário, em que o classificador somente indica se ocorreu um mal-posicionamento devido à rotação ou não, a média das taxas de Verdadeiros Positivos foram de 90\%, para todos os dedos, e $97 \%$, para o polegar somente. Na detecção do ângulo de rotação (segundo cenário), a média das taxas de Verdadeiros Positivos foram de 90\%, para todos os dedos, e $94 \%$, para o polegar somente.

Vale observar que o polegar possui uma área útil maior, e também a projeção em diferentes vistas produzem claramente larguras diferentes, desta forma permitindo que os resultados da classificação do polegar sejam melhores. As taxas de classificação demonstram claramente esta característica para a análise do polegar.

\section{CONCLUSÕES}

A avaliação da qualidade tradicional para sistemas baseados em contato com o sensor, proposta pelo NIST, não pode ser totalmente estendida e aplicada para sistemas de impressão digital sem contato com sensor utilizando múltiplas vistas. Os sistemas sem contato tem características específicas, devendo ainda ser consideradas para se estabelecer uma avaliação da qualidade da impressão digital adequada. O fator mais importante, que se deve considerar, é em relação à completa ausência de uma superfície de contato, permitindo com que durante o processo de captura, o dedo do usuário possua maior quantidade de graus de liberdade.

Este trabalho apresentou uma metodologia baseada na utilização de redes neurais artificiais, que permite a detecção do mal-posicionamento dos dedos na captura de impressões digitais para sistemas multivistas sem contato. A idéia principal desta proposta concentra-se na definição de um ponto 


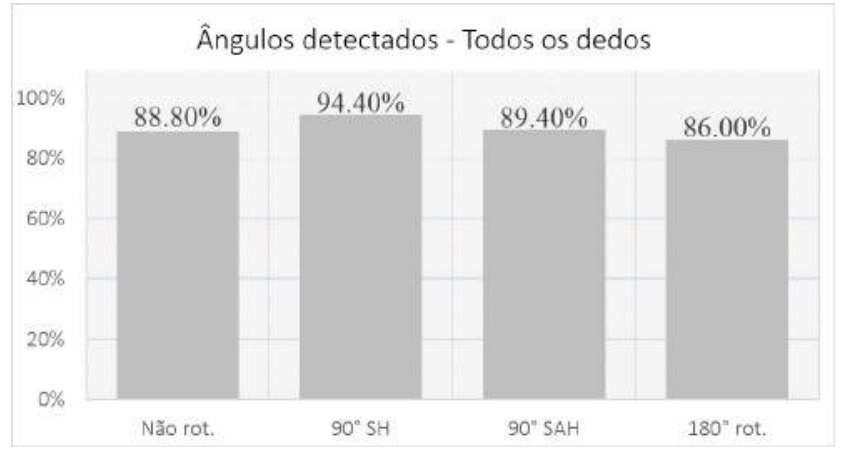

(a)

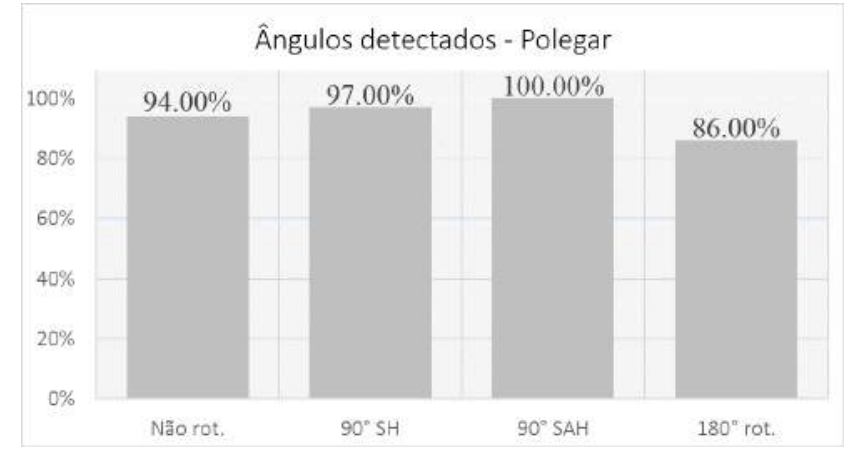

(b)

Figura 8: As porcentagens nas Tabelas 3 e 4 apresentam todos os resultados de acordo com os ângulos de rotação preditos. As diagonais que representam os verdadeiros positivos são apresentadas nos gráficos de barras em (a) e (b). A taxas médias de Verdadeiros Positivos em (a) e (b) são de $90 \%$ e $94 \%$, respectivamente.

Tabela 3: Resultado da detecção de ângulo de rotação: todos os dedos.

\begin{tabular}{|l|cccc|}
\multicolumn{5}{c}{ Predição } \\
\hline & Não rot. & $90^{\circ} \mathrm{SH}$ & $90^{\circ} \mathrm{SAH}$ & $180^{\circ}$ rot. \\
\hline \multirow{2}{*}{ Não rot. } & $88.80 \%$ & $0.50 \%$ & $0.40 \%$ & $10.30 \%$ \\
\hline $90^{\circ} \mathrm{SH}$ & $0.80 \%$ & $94.40 \%$ & $0.40 \%$ & $4.40 \%$ \\
\hline $90^{\circ} \mathrm{SAH}$ & $1.60 \%$ & $1.60 \%$ & $89.40 \%$ & $7.40 \%$ \\
\hline $180^{\circ}$ rot. & $9.70 \%$ & $3.40 \%$ & $0.90 \%$ & $86.00 \%$ \\
\hline
\end{tabular}

Tabela 4: Resultado da detecção de ângulo de rotação: polegar.

\begin{tabular}{|l|cccc|}
\multicolumn{5}{c}{ Predição } \\
\hline & Não rot. & $90^{\circ} \mathrm{SH}$ & $90^{\circ} \mathrm{SAH}$ & $180^{\circ}$ rot. \\
\hline \multirow{2}{*}{ Não rot. } & $94.00 \%$ & $2.50 \%$ & $0.50 \%$ & $3.00 \%$ \\
\hline $90^{\circ} \mathrm{SH}$ & $0.00 \%$ & $97.00 \%$ & $3.00 \%$ & $0.00 \%$ \\
\hline $90^{\circ} \mathrm{SAH}$ & $0.00 \%$ & $0.00 \%$ & $100.00 \%$ & $0.00 \%$ \\
\hline $180^{\circ}$ rot. & $3.50 \%$ & $7.00 \%$ & $3.50 \%$ & $86.00 \%$ \\
\hline
\end{tabular}

de partida para que as metodologias de avaliação de qualidade para este tipo de sistema possa ser formalizada.

Os resultados obtidos pela metodologia proposta alcançaram os seus objetivos, permitindo que fosse detectado o malposicionamento dos dedos e polegares com $93 \%$ e $97.25 \%$ dos casos, respectivamente. Esta metodologia considera cenários em que o classificador pode verificar o ângulo de rotação de todos os dedos, com média de acerto de $90 \%$ para os dedos e $94 \%$ para os polegares.

Reforça-se também que a metodologia proposta não faz uso de qualquer pré-processamento nas amostras avaliadas e nenhuma das amostras coletadas foi descartada na montagem da base de dados. Desta feita, a base de dados foi construída utilizando um conjunto de imagens heterogêneas com as mais variadas características de iluminação, translação, borramento e contraste. Sendo assim, ficou demonstrado que a metodologia proposta é robusta e capaz de lidar com situações complexas em que estes fatores implicariam na qualidade da imagem capturada, proporcionando resultados em que classificador utilizado fornece melhores resultados.

Em trabalhos futuros serão incluídos estudos de características complementares que permitam uma distinção mais refinada de detecção dos ângulos de rotação das imagens capturadas por este tipo de sensor. Além disso, outras métricas de qualidade podem ser consideradas e suas correlações com o mal-posicionamento rotacional dos dedos podem ser investigadas.

\section{REFERÊNCIAS}

[1] R. Allen, P. Sankar, and S. Prabhakar. Fingerprint identification technology. In J. Wayman, A. Jain, D. Maltoni, and D. Maio, editors, Biometric Systems: Technology, Design and Performance Evaluation, chapter 2. Springer, London, 2005.

[2] M. AlTarawneh, W. Woo, and S. Dlay. Objective fingerprint image quality assessment using gabor spectrum approach. In Digital Signal Processing, 2007 15th International Conference on, pages 248-251, July 2007.

[3] H. Demuth, M. Beale, H. Demuth, and M. Beale. Neural network toolbox for use with matlab, 1993.

[4] J. Dittmann and M. Hildebrandt. Context analysis of artificial sweat printed fingerprint forgeries: Assessment of properties for forgery detection. In Biometrics and Forensics (IWBF), 2014 International Workshop on, pages 1-6, March 2014.

[5] M. Dusio, M. A. Olsena, and C. Busch. Fingerprint sample quality assessment via ridge line count using laplacian of gaussian edge finding. In Biometrics and Forensics (IWBF), 2014 International Workshop on, pages 1-6, March 2014.

[6] J. Hammerle-Uhl, M. Pober, and A. Uhl. Systematic evaluation methodology for fingerprint-image quality assessment techniques. In Information and Communication Technology, Electronics and Microelectronics (MIPRO), 2014 37th International Convention on, pages 1315-1319, May 2014.

[7] S. Haykin. Neural Networks: A Comprehensive Foundation. Prentice Hall PTR, Upper Saddle River, NJ, USA, 2nd edition, 1998.

[8] A. K. Jain and S. Pankanti. Automated fingerprint identification and imaging systems. In H. C. Lee and R. E. Gaensslen, editors, Advances in Fingerprint Technology, chapter 8. CRC Press, Boca Raton, 2nd edition, 2001.

[9] R. Khanna and W. Shen. Automated fingerprint identification system (afis) benchmarking using the national institute of standards and technology (nist) special database 4. In Security Technology, 1994. Proceedings. 
Institute of Electrical and Electronics Engineers 28th Annual 1994 International Carnahan Conference on, pages 188-194, Oct 1994.

[10] A. Kumar and C. Kwong. Towards contactless, low-cost and accurate $3 \mathrm{~d}$ fingerprint identification. In Computer Vision and Pattern Recognition (CVPR), 2013 IEEE Conference on, pages 3438-3443, June 2013.

[11] R. Labati, V. Piuri, and F. Scotti. Neural-based quality measurement of fingerprint images in contactless biometric systems. In Neural Networks (IJCNN), The 2010 International Joint Conference on, pages 1-8, july 2010.

[12] R. D. Labati, V. Piuri, and F. Scotti. A neural-based minutiae pair identification method for touch-less fingerprint images. In Computational Intelligence in Biometrics and Identity Management (CIBIM), 2011 IEEE Workshop on, pages 96 -102, april 2011.

[13] F. Liu, D. Zhang, C. Song, and G. Lu. Touchless multiview fingerprint acquisition and mosaicking. Instrumentation and Measurement, IEEE Transactions on, 62(9):2492-2502, Sept 2013.

[14] D. Milgram. Computer methods for creating photomosaics. IEEE Transactions on Computers, C-24(11):1113-1119, Nov. 1975.

[15] M. Olsen, E. Tabassi, A. Makarov, and C. Busch. Self-organizing maps for fingerprint image quality assessment. In Computer Vision and Pattern Recognition Workshops (CVPRW), 2013 IEEE Conference on, pages 138-145, June 2013.

[16] S. Pankanti, S. Prabhakar, and A. Jain. On the individuality of fingerprints. Pattern Analysis and Machine Intelligence, IEEE Transactions on, 24(8):1010-1025, Aug 2002.

[17] G. Parziale. Touchless fingerprinting technology. In N. K. Ratha and V. Govindaraju, editors, Advances in Biometrics: Sensors, Algorithms and Systems, chapter 2. Springer, London, 2008.

[18] V. Piuri and F. Scotti. Fingerprint biometrics via low-cost sensors and webcams. In Biometrics: Theory, Applications and Systems, 2008. BTAS 2008. 2nd IEEE International Conference on, pages 1-6, 29 2008-oct. 12008.

[19] J. Wayman. A definition of biometrics. In National Biometric Test Center Colected Works 1997-2000. San Jose State University, 2000.

[20] B. Wing. Nist contributions to biometric technology. IT Professional, 16(2):38-44, Mar 2014.

[21] J.-H. Xue and D. Titterington. t -tests, f -tests and otsu's methods for image thresholding. Image Processing, IEEE Transactions on, 20(8):2392-2396, Aug 2011.

[22] H. Yu and B. M. Wilamowski. Levenberg-marquardt training. In Industrial Electronics Handbook, 2nd Edition, volume 5, pages 12-1. CRC Press, 2011. 
XI Brazilian Symposium on Information System, Goiânia, GO, May 26-29, 2015. 